\title{
Subcutaneous Cheek Nodule Associated with Granulomatosis with Polyangiitis
}

\author{
Makiko Hayashi ${ }^{1}$, Shinjiro Kaieda ${ }^{1}$, Aya Kawaguchi ${ }^{2,3}$, Masahiro Tsutsumi ${ }^{3}$, Yumi Harada ${ }^{1}$, \\ Takuma Koga ${ }^{1}$, Jun Akiba ${ }^{4}$, Tomoaki Hoshino ${ }^{1}$ and Hiroaki Ida ${ }^{1}$
}

\begin{abstract}
:
We herein report an unusual case of granulomatosis with polyangiitis (GPA) in a 65-year-old man in whom relapsed disease manifested as an anterior cheek nodule. Magnetic resonance imaging indicated the differential diagnoses of the subcutaneous nodule in the patient's anterior cheek to be inflammatory granulomatous lesions with GPA, malignancy, or infectious disease. A histopathological examination ruled out malignancy and infectious diseases, and necrotizing vasculitis was suspected. The subcutaneous nodule was successfully treated using rituximab, suggesting that it was associated with GPA, secondary to vasculitis. Clinicians should be aware of the possibility of such a rare manifestation of GPA.
\end{abstract}

Key words: granulomatosis with polyangiitis, rituximab, subcutaneous anterior cheek nodule

(Intern Med 60: 3823-3826, 2021)

(DOI: 10.2169/internalmedicine.6541-20)

\section{Introduction}

Granulomatosis with polyangiitis (GPA) is an autoimmune vasculitis characterized primarily by necrotizing granulomatous lesions of the upper and lower respiratory tract and kidney (1). In addition to this classical clinical triad, GPA can affect other tissues, such as the dura mater and juxtavertebral region $(2,3)$. The prevalence of specific cutaneous lesions in GPA has been reported to be 34\% (223 of 656 of participants) (4). The most frequently encountered skin manifestations were petechiae or purpura (16\%), followed by painful skin lesions (9.4\%) and maculopapular rash $(6.7 \%)$. Non-tender skin nodules associated with GPA were also reported, but these were rare (1.1\%) (4). A small case series of patients with cutaneous lesions associated with GPA included individuals with palpable purpura (8 of 21 , $38 \%)$, mucocutaneous ulcers $(6 / 21,29 \%)$, and subcutaneous nodules $(3 / 21,14 \%)(5)$. Thus, subcutaneous involvement of GPA is uncommon.

We herein report a very rare case of GPA that was associated with an anterior cheek nodule and successfully treated using rituximab.

\section{Case Report}

A 61-year-old man presented with arthralgia, numbness in both upper extremities, and hypacousia in December 2016.

His height was $166.7 \mathrm{~cm}$, and his body mass was $83.6 \mathrm{~kg}$. His vital signs were normal, but he had hypertension and chronic paranasal sinusitis. Head and chest computed tomography revealed no abnormalities. An examination by an otolaryngologist identified a left inner-ear disorder, and an examination by a neurologist identified bilateral median nerve and right ulnar nerve palsy, suggesting the presence of mononeuritis multiplex.

His proteinase-3 anti-neutrophil cytoplasmic antibody (PR 3-ANCA) titer was high (116.1 IU/L; normal titer $<3.5 \mathrm{IU} /$ L). The patient met the criteria for a clinical diagnosis of ANCA-associated vasculitis based on the presence of PR3ANCA and mononeuritis multiplex (6). A diagnosis of GPA was made in accordance with Watts' algorithm because diagnostic surrogate markers of GPA, such as chronic sinusitis and mastoiditis, had been present for at least three months,

\footnotetext{
${ }^{1}$ Department of Medicine, Division of Respirology, Neurology and Rheumatology, Kurume University School of Medicine, Japan, ${ }^{2}$ Department of Pathology, Kurume University School of Medicine, Japan, ${ }^{3}$ Department of Dermatology, Kurume University School of Medicine, Japan and ${ }^{4}$ Department of Diagnostic Pathology, Kurume University Hospital, Japan
}

Received for publication October 17, 2020; Accepted for publication April 7, 2021

Correspondence to Dr. Shinjiro Kaieda, shinkaieda@gmail.com 
and serology was positive for PR3-ANCA (6). The GPA did not affect his lungs or kidneys, suggesting that it was limited to his upper airway.

Outpatient therapy with oral prednisolone $30 \mathrm{mg}$ daily was initiated in February 2017, and the arthralgia and mononeuritis multiplex disappeared, although the patient's hearing acuity deteriorated. Although life-threatening organ damage was not present at the onset of the disease, the hearing loss was progressive, and his PR3-ANCA titer was high at the initial diagnosis. Relapses of ANCA-associated vasculitis have been reported to occur more frequently in patients who are PR3-ANCA-positive than in those who are myeloperoxidase (MPO)-ANCA-positive $(7,8)$. Therefore, oral treatment with cyclophosphamide $(50 \mathrm{mg} /$ day $)$ was added to

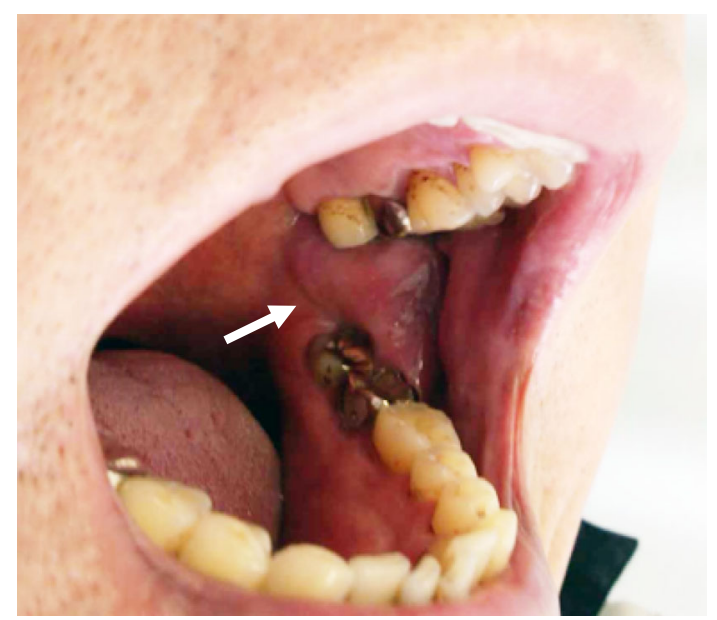

Figure 1. Clinical presentation of the patient at the relapse of disease. A soft tissue mass lesion was observed in the oral cavity (arrow). the steroid therapy; however, this was subsequently discontinued because of dizziness after 3 months. Azathioprine 50 $\mathrm{mg} /$ daily was also administered for 6 weeks, but this was also discontinued because of liver toxicity. Therefore, methotrexate (MTX: $6 \mathrm{mg} / \mathrm{week}$ ) treatment was initiated in October 2017, and the dose was increased to $10 \mathrm{mg} / \mathrm{week}$ after 10 months. However, the MTX was also discontinued because its therapeutic effect was unclear and because of high serum liver enzyme activities. Rituximab (RTX) was then administered at $730 \mathrm{mg}\left(500 \mathrm{mg} / \mathrm{m}^{2}\right)$ weekly for 4 weeks, starting in July 2018, which ameliorated the patient's sinusitis and was not associated with further deterioration in his hearing acuity. Steroid monotherapy was continued after the RTX therapy was complete, as other immunosuppressants, including cyclophosphamide, azathioprine, and MTX, could not be used in this patient.

In January 2020, at 65 years old, the patient noted a swelling in his left cheek and a non-tender mass in his oral cavity (Fig. 1, arrow). The dose of prednisolone was reduced to $11 \mathrm{mg} / \mathrm{day}$, and laboratory investigations revealed a white blood cell count of $7,000 / \mathrm{mm}^{3}$ (70.5\% neutrophils), a hemoglobin level of $15.2 \mathrm{~g} / \mathrm{dL}$, and a platelet count of $345,000 /$ $\mathrm{mm}^{3}$, along with normal routine blood chemistry and coagulo-fibrinolytic test results. The patient's C-reactive protein (CRP) concentration was slightly high $(0.2 \mathrm{mg} / \mathrm{dL}$; normal concentration $<0.14 \mathrm{mg} / \mathrm{dL}$ ). A urinalysis was negative for urinary protein, and no casts were observed in the urinary sediment. Immunological testing showed that his PR3ANCA titer was elevated (45.5 IU/L), but his serum MPOANCA titers were within the normal ranges. The laboratory findings are shown in the Table.

The patient's right maxillary sinusitis and the subcutaneous nodule in his left anterior cheek were indicated as high-

Table. Laboratory Data.

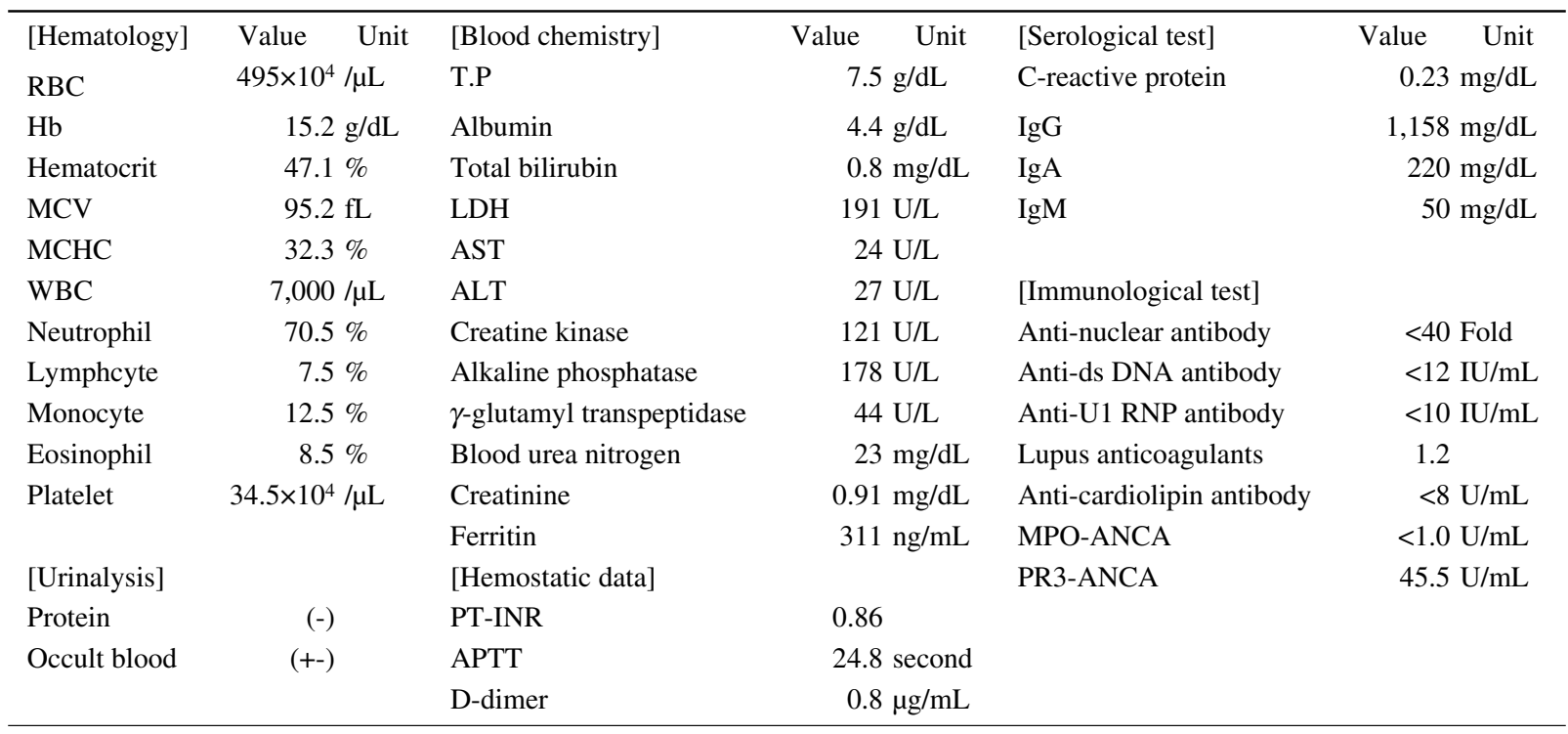

RBC: red blood cells, MCV : mean corpuscular volume, MCHC: mean corpuscular hemoglobin concentration, T.P: total protein, LDH: lactate dehydrogenase, AST: aspartate transaminase, ALT: alanine transaminase, PT-INR: prothrombin time/international normalized ratio, APTT: activated partial thromboplastin time, Anti-ds: Anti-double-stranded, RNP: ribonucleoprotein, MPO-ANCA: myeloperoxidase-anti-neutrophil cytoplasmic antibody, PR3-ANCA: proteinase 3-anti-neutrophil cytoplasmic antibody 


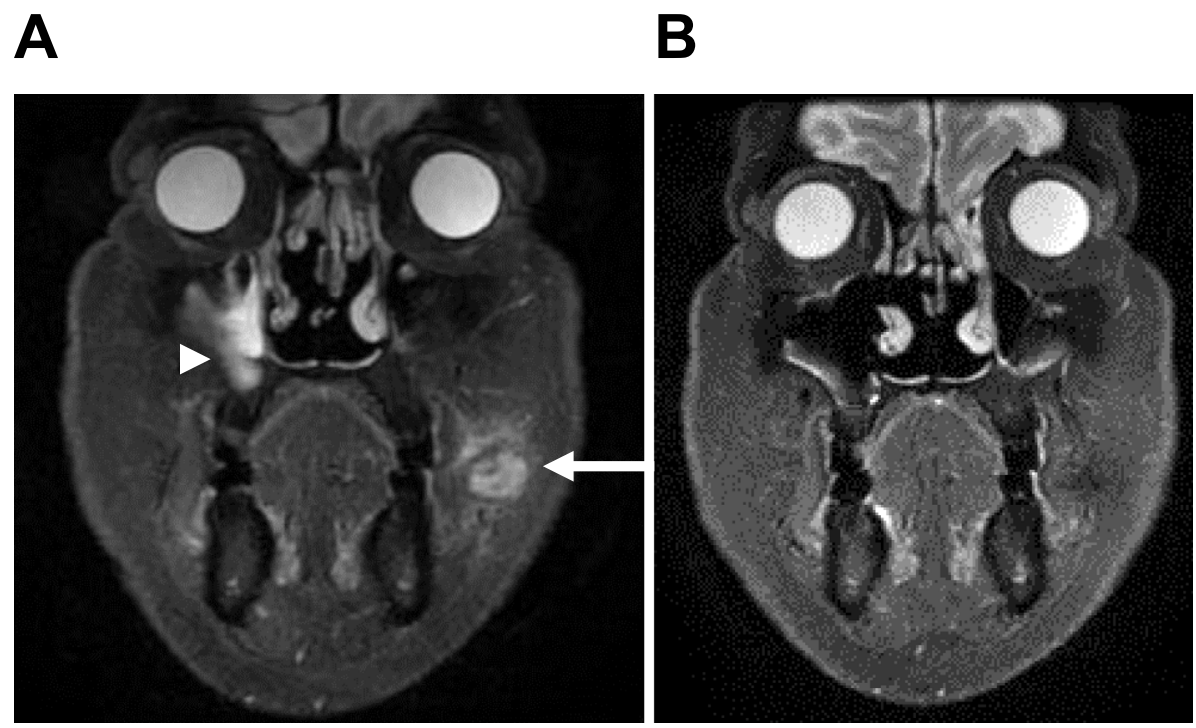

Figure 2. T2-weighted fluid-attenuated inversion recovery magnetic resonance images obtained before (A) and after (B) rituximab therapy. Right maxillary sinusitis (arrowhead) and a subcutaneous nodule (arrow) were identified (A). After rituximab therapy, the sinusitis had improved, and the subcutaneous nodule had shrunk (B).

A

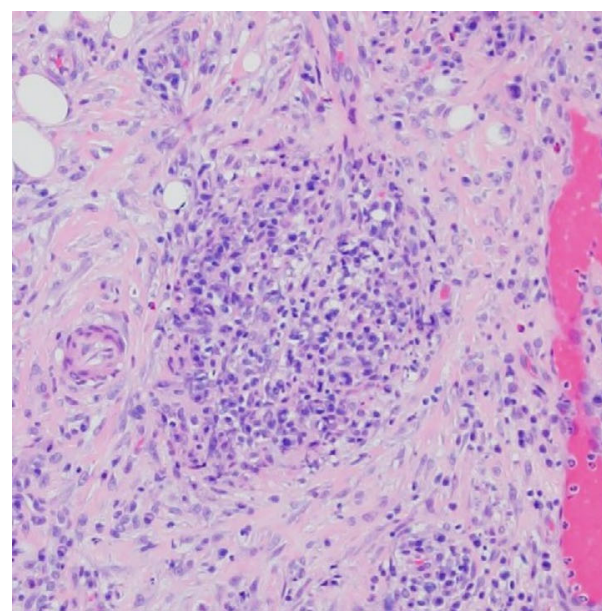

B

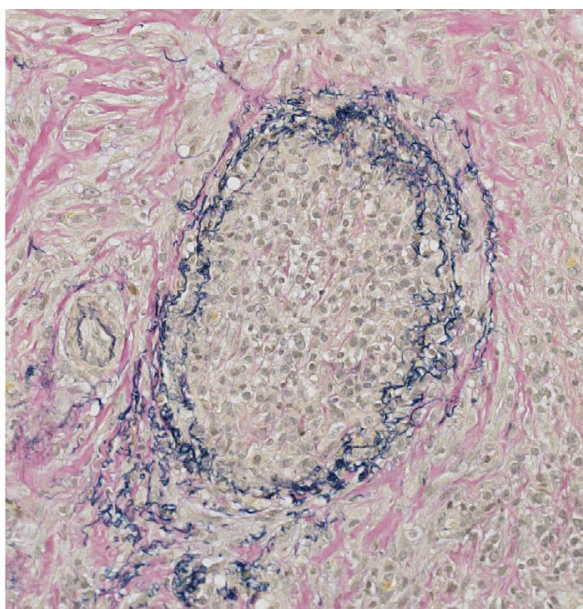

Figure 3. Results of a skin biopsy revealing necrotizing vasculitis. A: Diffuse infiltration of inflammatory cells around the blood vessels (Hematoxylin and Eosin staining; original magnification $\times 400$ ). B: The elastic laminae are partially obscured (Elastica van Gieson stain; original magnification $\times 400$ ).

intensity lesions using T2-weighted fluid-attenuated inversion recovery magnetic resonance imaging (MRI) (Fig. 2A). The buccinator muscle surrounding the subcutaneous cheek nodule also showed a relatively high intensity. T1-weighted MRI showed these lesions to have low intensity. Therefore, the differential diagnoses of the subcutaneous nodule in the patient's anterior cheek were inflammatory granulomatous lesions with GPA, malignancy, and infectious disease.

A percutaneous biopsy of the subcutaneous nodule was performed via the anterior cheek, and a histopathological assessment revealed diffuse infiltration of inflammatory cells around the blood vessels (Fig. 3A). Although fibrinoid necrosis was not apparent, the elastic laminae were partially obscured (Fig. 3B). Based on these histopathological findings, necrotizing vasculitis was suspected. In addition, malignancy and infectious diseases were ruled out. Therefore, RTX therapy was repeated, and the patient's maxillary sinusitis and subcutaneous nodule improved (Fig. 2B). There were no adverse effects of RTX therapy, including no cytopenia or infection. The patient's CRP returned to normal, but his PR3-ANCA titer remained high at $49.1 \mathrm{IU} / \mathrm{L}$.

The patient provided his written informed consent for the inclusion of his clinical data in this report, and the investigations were performed according to the Declaration of Helsinki. Ethics approval was obtained from the Institutional Review Board of our hospital. 


\section{Discussion}

In the present case, the patient met the criteria for a clinical diagnosis of ANCA-associated vasculitis based on the presence of PR3-ANCA and mononeuritis multiplex. Sinusitis was not detected using computed tomography at his first visit to the outpatient department, but right maxillary sinusitis was confirmed using MRI when he developed a subcutaneous nodule in his cheek (Fig. 2A). Although mastoiditis was not detected on imaging, the patient's hearing loss was progressive, and an otolaryngologist identified a left innerear disorder. Therefore, we diagnosed him with GPA, in accordance with Watts' algorithm. The patient developed subcutaneous nodules in his left cheek and this was suspected of being a complication of GPA; however, MRI suggested the possibility of malignancy or infectious diseases. Therefore, a histopathological assessment was needed to confirm the nature of the subcutaneous nodule in his anterior cheek. The histopathological examination ruled out malignancy and infectious diseases, and necrotizing vasculitis was suspected. Therefore, RTX therapy was repeated, and the subcutaneous nodule improved. As a consequence, the subcutaneous nodule was considered to have been induced by GPA, owing to necrotizing vasculitis.

Subcutaneous nodules associated with GPA have previously been reported in $1.1 \%-14.21 \%$ of patients with GPA $(4,5)$. These nodules are reportedly located in the back, right armpit, and forehead of patients with GPA (5), whereas subcutaneous lesions in the anterior cheek have very rarely been reported (9). Shenavandeh et al. reported the involvement of GPA in a patient who developed severe sinusitis, hearing loss, and a left cheek mass. They reported that the left cheek mass was caused by osteomyelitis of the maxillary bone, based on their MRI findings, but a histopathological analysis was not performed. Treatment with steroids and oral cyclophosphamide resolved the left cheek mass in this case. We recommend that clinicians bear in mind the possibility that GPA may rarely manifest in this way.

In summary, we encountered a very rare case of GPA that was associated with an anterior cheek nodule, which developed secondary to necrotizing vasculitis. However, the iden- tification of similar cases is required to facilitate the elucidation of the mechanism whereby GPA manifests subcutaneously.

The authors state that they have no Conflict of Interest (COI).

\section{Acknowledgement}

We thank Mark Cleasby, PhD, for editing a draft of this manuscript.

\section{References}

1. Hoffman GS, Kerr GS, Leavitt RY, et al. Wegener granulomatosis: an analysis of 158 patients. Ann Intern Med 116: 488-498, 1992.

2. Kaieda S, Yoshida N, Minezaki M, et al. The Successful treatment of myeloperoxidase antineutrophil cytoplasmic antibody-positive hypertrophic pachymeningitis in patients with the limited form of granulomatosis with polyangiitis using methotrexate: two case reports. Intern Med 56: 959-965, 2017.

3. Yoshida Y, Kaieda S, Furuta T, Ida H. Juxta-vertebral lesions associated with granulomatosis with polyangiitis. Intern Med 58: 25872588, 2019.

4. Micheletti RG, Chiesa Fuxench Z, Craven A, et al. Cutaneous manifestations of antineutrophil cytoplasmic antibody-associated vasculitis. Arthritis Rheumatol 72: 1741-1747, 2020.

5. Montero-Vilchez T, Martinez-Lopez A, Salvador-Rodriguez L, et al. Cutaneous manifestations of granulomatosis with polyangiitis: a case series study. Acta Derm Venereol 100: adv00150, 2020.

6. Watts R, Lane S, Hanslik T, et al. Development and validation of a consensus methodology for the classification of the ANCAassociated vasculitides and polyarteritis nodosa for epidemiological studies. Ann Rheum Dis 66: 222-227, 2007.

7. Lionaki S, Blyth ER, Hogan SL, et al. Classification of antineutrophil cytoplasmic autoantibody vasculitides: the role of antineutrophil cytoplasmic autoantibody specificity for myeloperoxidase or proteinase 3 in disease recognition and prognosis. Arthritis Rheum 64: 3452-3462, 2012.

8. Charles P, Perrodeau E, Samson M, et al. Long-term rituximab use to maintain remission of antineutrophil cytoplasmic antibodyassociated vasculitis: a randomized trial. Ann Intern Med 173: 179-187, 2020.

9. Shenavandeh S, Petramfar P. Three atypical manifestations of granulomatosis with polyangiitis: lateral medullary syndrome, anterior cheek mass and melting scleritis of eye. Reumatologia 55: 145-150, 2017.

The Internal Medicine is an Open Access journal distributed under the Creative Commons Attribution-NonCommercial-NoDerivatives 4.0 International License. To view the details of this license, please visit (https://creativecommons.org/licenses/ by-nc-nd/4.0/).

\section{(C) 2021 The Japanese Society of Internal Medicine} Intern Med 60: 3823-3826, 2021 